\title{
Reduced away-from-home food expenditure and better nutrition knowledge and belief can improve quality of dietary intake among US adults
}

\author{
May A Beydoun ${ }^{1}$, Lisa M Powell ${ }^{2}$ and Youfa Wang ${ }^{1, *}$ \\ ${ }^{1}$ Center for Human Nutrition, Department of International Health, Johns Hopkins Bloomberg School of Public \\ Health, 615 N Wolfe Street E2546, Baltimore, MD 21205, USA: ${ }^{2}$ Institute for Health Research and Policy, \\ University of Illinois at Chicago, Chicago, IL, USA
}

Submitted 20 February 2007: Accepted 25 February 2008: First published online 22 April 2008

\begin{abstract}
Objective: To test whether reduced away-from-home food expenditure (AFHFE) and better nutrition knowledge and beliefs (NKB) are associated with dietary quality among US adults.

Design and subjects: The dietary intake data (average of two $24 \mathrm{~h}$ recalls) used were collected from US adults (20-65 years) participating in two cross-sectional surveys, the 1994-96 Continuing Survey of Food Intake by Individuals (CSFII; $n$ 7148) and the CSFII/Diet and Health Knowledge Survey (DHKS; $n$ 4252).

Outcome measures: Dietary quality was assessed using selected nutrients and food groups and the 2005 revised US Department of Agriculture Healthy Eating Index (HEI).

Exposure variables: (i) Absolute AFHFE (weekly, per capita) and proportion of this exposure out of total food expenditure (relative expenditure); (ii) NKB score using a composite of an eleven-item scale elicited among the CSFII/DHKS subgroup.

Statistical analyses performed: We used $t$ tests, $\chi^{2}$ tests, Wilcoxon rank-sum tests and multivariate linear regression models adjusting standard errors for sample design complexity. We utilized a change-in-estimate approach to assess mediation. For effect modification, we tested the significance of interaction terms $(\mathrm{NKB} \times \mathrm{AFHFE})$.

Results: Absolute AFHFE was positively associated with grams of fat $(\beta=0 \cdot 14$ (SE $0 \cdot 06))$ and saturated fat $(\beta=0.02(\operatorname{SE} 0 \cdot 01))$ and negatively associated with fibre $(\beta=-0 \cdot 02(\operatorname{se~} 0 \cdot 01))$ and HEI $(\beta=-0 \cdot 08(\operatorname{se~} 0 \cdot 01))$. Relative AFHFE mediated NKB effects on intakes such as fat, saturated fat, cholesterol, $\mathrm{Na}$, and fruits and vegetables (change in estimate $>10 \%$ ). Among subjects with a poor NKB score, higher AFHFE resulted in lower diet quality, particularly $\mathrm{Na}$ and cholesterol intakes.

Conclusions: Higher AFHFE was associated with a lower dietary quality and interacted antagonistically with $\mathrm{NKB}$ in some instances, while mediating the relationship between $\mathrm{NKB}$ and dietary quality in others.
\end{abstract}

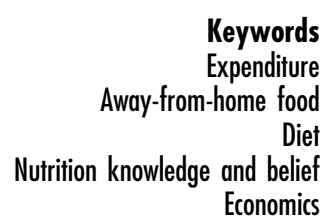

The risk of obesity and other chronic conditions may be alleviated by improving diet quality ${ }^{(1)}$, which in turn may be influenced by the site of food preparation and consumption. The percentage of spending on foods away from home in the American diet has risen from 25\% in 1970 to $40 \%$ in $1995^{(2)}$. Its concomitance with the rising obesity epidemic may suggest a causal association that is mediated by poorer diet quality among Americans. Indeed, parallel to the rising obesity epidemic, food consumption patterns and household expenditures both show a marked upward trend in total energy intake derived from away-from-home sources. In 2002, nearly half of Americans' food expendi- tures went to an away-from-home food facility. Total awayfrom-home food expenditures were \$US 415 billion in 2002, up 58\% (23\% in constant dollars) from \$US 263 billion in $1992^{(3)}$. Categorizing energy intake from food source locations separately by home, restaurants, fast-food establishments, schools/day care and other non-home locations, researchers have reported significant increases in the last few decades in the proportion of food prepared away from home with particularly high increases in fast-food consumption. The percentage of total energy intake from food prepared away from home increased from $18 \%$ in 1977-1978 to $32 \%$ in 1994-1996, and the percentage of 
energy from fast food increased threefold for adults aged 18 years and over and fivefold for children aged 2-17 years ${ }^{(4)}$. Examining similar trends, another study showed that the proportion of total energy coming from restaurant and fastfood places combined tripled among adolescents (aged 12-18) and doubled among young adults (aged 19-29) between 1977 and 1996 $6^{(5)}$. Similar dietary behaviour changes were observed for other age groups as well ${ }^{(6)}$.

While previous studies have suggested a negative influence of fast-food and away-from-home food consumption on dietary quality, BMI and obesity among all age groups ${ }^{(7-10)}$, to our knowledge no previous research has explored the direct effect of household away-fromhome food expenditure (AFHFE) on the quality of dietary intake among individuals. The present study aimed to assess the nutritional impact of household expenditure on food bought and eaten away from home, such as at restaurants and fast-food places, and whether it is affected by people's nutrition knowledge and beliefs (NKB).

Several hypotheses were tested using nationally representative data. We were particularly interested in the effect of expenditure on several measures of dietary quality (Fig. 1, $\mathrm{H}_{2}$ ). As a first analysis, we examined sociodemographic, economic and lifestyle correlates of AFHFE (Fig. 1, $\mathrm{H}_{1}$ ). Furthermore, knowledge and beliefs, acquired through education and other cultural and social support mechanisms, may influence food purchasing and in turn affect dietary intake (Fig. 1, $\mathrm{H}_{4}$ ). We hypothesized that individuals with stronger NKB (i.e. nutrition is important to them) have reduced AFHFE (Fig. 1, $\mathrm{H}_{3}$ ), which improves diet quality. Hence, AFHFE may act as a mediator (Fig. 1, $\mathrm{H}_{5}$ ). In addition, NKB may actually moderate the effect of expenditure on dietary quality, by acting as an effect modifier (Fig. 1, $\mathrm{H}_{6}$ ). In particular, we hypothesized that increased household AFHFE reduces individual diet quality mostly among subjects with low NKB scores. Finally, we looked at $\mathrm{NKB}$ as a potential confounder in the relationship between expenditure and diet quality (Fig. 1, $\mathrm{H}_{7}$ ).

\section{Data and methods}

\section{Survey methods}

Data from the US Department of Agriculture's (USDA) Continuing Survey of Food Intakes by Individuals (CSFII) 1994-96 were used ${ }^{(11)}$. This is a nationally representative, multistage, stratified sample of 16103 non-institutionalized persons aged 0 to 90 years residing in the USA containing information about dietary intake (by one or two nonconsecutive, multiple-pass, $24 \mathrm{~h}$ recalls that were 3 to $10 \mathrm{~d}$ apart), socio-economic and demographic parameters. In addition, a sample of subjects who responded to at least the first day of the two $24 \mathrm{~h}$ recalls in CSFII (one adult aged 20 years or older per household) completed the Diet and Health Knowledge Survey (DHKS) in which they answered questions related to knowledge and beliefs

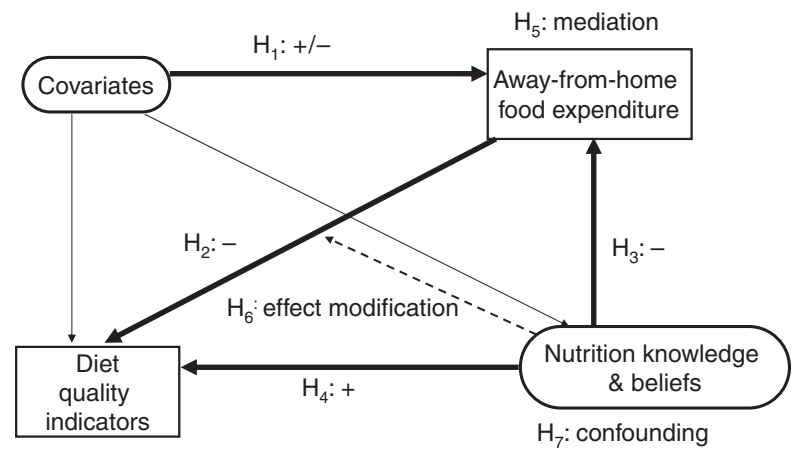

Fig. 1 Hypotheses tested in the present study. Notation: + , positive association; - , negative association; $\mathrm{H}_{1}$, hypothesis that covariates are associated with away-from-home food expenditure (AFHFE); $\mathrm{H}_{2}$, hypothesis that AFHFE is negatively associated with diet quality indicators and new Healthy Eating Index $(\mathrm{HEI}) ; \mathrm{H}_{3}$, hypothesis that nutrition knowledge and beliefs (NKB) is negatively associated with AFHFE; $\mathrm{H}_{4}$, hypothesis that NKB is positively associated with diet quality indicators and new $\mathrm{HEl} ; \mathrm{H}_{5}$, hypothesis that the effect of NKB on diet quality indicators and new $\mathrm{HEI}$ is mediated by AFHFE; $\mathrm{H}_{6}$, hypothesis that the effect of AFHFE on diet quality indicators is modified by NKB; $\mathrm{H}_{7}$, hypothesis that the effect of AFHFE on diet quality indicators is confounded by NKB

about diet and health. Demographic, socio-economic and lifestyle variables were available for all individuals who participated in the CSFII. Although this data set is 10 years old, it is the only available data set that provides comprehensive, nationally representative data on all the study variables needed in the present project, such as detailed information on participants' nutrition knowledge, beliefs and perceptions, which are not collected in other more recent national surveys such as the National Health and Nutrition Examination Surveys ${ }^{(12)}$.

\section{Study sample}

Among the 16103 respondents who completed CSFII 1994-96, 9872 who were 20 years or older had data on day 1 of recall. Out of those, only 5765 subjects (one person per household) were sampled as respondents to the DHKS. We further excluded those over the age of 65 years ( $n 2127$ for CSFII, $n 1319$ for CSFII/DHKS) and those who completed only one $24 \mathrm{~h}$ dietary recall ( $n 414$ for CSFII, $n 90$ for CSFII/DHKS) or did not provide data on AFHFE ( $2 \%$ and $4 \%$ missing in each sample, respectively). As a result, our final sample consisted of 7148 (3625 men and 3523 women) individuals among those who completed CSFII for the time period 1994-1996 (i.e. study sample 1) and 4252 individuals (2164 men and 2088 women) who completed both surveys (CSFII and DHKS, i.e. study sample 2).

\section{Measures}

Food expenditure assessment

The CSFII 1994-96 interviews asked one respondent from each household to estimate the average weekly amount 
of dollars spent on food while shopping at various types of outlets for all household members. Food outlet type was broadly classified as: (i) grocery stores (including the store's salad bar, soup bar and delicatessen); (ii) specialty stores (including bakeries, liquor stores, delicatessens, meat markets, vegetable stands, health food stores and other similar places); (iii) fast-food or carryout places (when food was consumed at home); and (iv) away-fromhome foods (including food and beverages that never entered the home, i.e. eaten at restaurants, fast-food places, cafeterias at work or at school, or purchased from vending machines).

The main exposure of interest is the weekly dollars spent per capita on away-from-home food (i.e. 'absolute expenditure'). This measure was computed by first standardizing responses to weekly expenditures as some were reported on a monthly basis. Second, we divided that standardized measure by household size to obtain per capita absolute AFHFE. However, 'relative expenditure' on away-from-home food was also considered in comparison with total expenditure on food (i.e. the proportion $=($ AFHFE $/$ total food expenditure $) \times 100 \%)$. Total food expenditure was measured by adding all four amounts ((i) to (iv) mentioned earlier) after standardizing responses to weekly expenditures and subtracting nonfood expenditures as reported by a fifth question related to grocery expenses. Appendix 1 presents the household questionnaire items used to derive the two expenditure variables. Note that while away-from-home foods refer to the foods themselves (either in servings or in grams), AFHFE is the money spent by the household on foods purchased and consumed outside the home environment.

\section{Dietary intake and diet quality indicators}

Dietary intake was elicited from subjects participating in the CSFII 1994-96 survey using two $24 \mathrm{~h}$ dietary recalls. Based on responses which uncovered types of foods consumed during these two days along with their portion sizes, nutrient intakes were provided based on food composition tables that were designed specifically to be used for the survey. Moreover, the foods consumed were grouped into broader categories. Average dietary intakes of foods and nutrients from the two $24 \mathrm{~h}$ recalls were considered. Several indicators and indices were created accordingly, as measures of diet quality. These included the intake of total fat and saturated fat (both in grams and as a percentage of total energy), dietary cholesterol (mg), $\mathrm{Na}$ (mg), added sugars (teaspoons) and sweetened beverages (grams), the excess of which are expected to reduce diet quality. We also considered intake of fruits and vegetables (VF in servings), dairy products (servings) and fibre (grams), adequate intakes of which improve diet quality.

To assess the overall quality of the diet, we applied the USDA's Healthy Eating Index (HEI). While this index has been used in its earlier version ${ }^{(13)}$ so far in the literature, we used the new HEI 2005 described in detail in Appendix $2^{(14)}$. The HEI was revised to reflect the 2005 Dietary Guidelines for Americans and had a number of improvements $^{(14)}$. The new HEI includes twelve components and is measured on a scale of 0 to 100 . For many of the food group criteria described in Appendix 2, serving estimates rather than grams were used as made available by the USDA.

\section{Correlates of away-from-bome food expenditure}

We tested the association between several household-level and geographic-level variables with household AFHFE. These included mean age in the household, number of children under the age of 18 years in each household, percentage female, percentage non-white based on the race/ethnicity variable (non-Hispanic whites or Caucasians; non-Hispanic blacks or African Americans; Hispanics; all others such as Asians, Native Americans, Pacific Islanders and Alaskan Natives), 1990 Census geographic regions (north-east; mid-west; south; west), degree of urbanization of the geographical area in which the household is located (Metropolitan Statistical Area (MSA)-central city; MSAsuburban; rural), mean educational attainment of adults over the age of 20 years ( $<$ high school, 1-8 years; high school, 9-12 years; $>$ high school, $\geq 13$ years) in the household, household annual income per capita (total annual income divided by household size expressed in \$US) and current employment status (employed; not employed).

In addition, we considered individual-level lifestyle factors. These included current smoking status ( $1=$ yes; $2=$ no) and physical exercise (scale of 1 to 6 on the following question: 'How often do you exercise vigorously enough to work up a sweat?', with $1=$ daily and $6=$ rarely or never). Sedentary lifestyle was operationalized as those whose response was 'rarely or never'. Individual-level age, gender, race, education, employment status, smoking status and sedentary behaviour were considered as potential confounders in all multivariate analyses. Moreover, we considered individuallevel food purchase factors as potential confounders based on the question: "When buying a food, what is important to you?' and included taste, cost, convenience, safety, and how well food keeps. Each variable was on a scale of $1=$ not important at all to $4=$ very important.

\section{Nutrition knowledge and beliefs}

One adult survey participant from each household was asked about his diet and health-related knowledge and beliefs in the CSFII/DHKS. We examined one DHKS scale consisting of eleven questions which were initiated by the following cue: 'To you personally, is it very important (score of 4), somewhat important (3), not too important (2), or not at all important (score of 1) to...' (i) use salt or sodium only in moderation? (ii) Choose a diet low in saturated fat? (iii) Choose a diet with plenty of VF? 
(iv) Use sugars only in moderation? (v) Choose a diet with adequate fibre? (vi) Eat a variety of foods? (vii) Maintain a healthy weight? (viii) Choose a diet low in fat? (ix) Choose a diet low in cholesterol? (x) Choose a diet with plenty of breads, cereals, rice and pasta? (xi) Eat at least two servings of dairy products daily? It is worth noting that eight of the eleven NKB questions reflected the diet quality indicators considered as outcomes.

Using principal components analysis (PCA) ${ }^{(15)}$, we created an index by extracting a single component score by imposing an eigenvalue criterion $>1 \cdot 0$, as well as examining the scree plot. The component explained around $40 \%$ of the variance in the eleven items, which loaded almost equally on this component (loadings ranged from 0.23 and 0.35 ). The score was estimated and subdivided into tertiles in part of the analysis. Those who were in the lowest tertile were considered to have poor NKB.

\section{Statistical analyses}

Two-way frequencies and descriptive statistics were calculated for several covariates (demographic, geographic, socio-economic, lifestyle and NKB) by AFHFE (absolute and relative) quintiles; proportions (\%) were calculated for categorical variables; and means with their standard errors were computed for continuous variables. For hypothesis testing regarding the equality of means between groups, we performed $t$ tests. For testing the association between categorical variables, we conducted $\chi^{2}$ tests. Furthermore, we performed a non-parametric test for trend in means of continuous variables across ordered groups (e.g. quintiles of expenditure) using a test developed by Cuzick ${ }^{(16)}$, which is an extension of the Wilcoxon rank-sum test, correcting for ties. Note that these tests could not take into account effects of the complex sampling design, although it is unlikely that the conclusions would be changed if they could.

Multivariate linear regression models were used to examine the relationship between the main exposure variables (expenditure, continuous) and diet quality indicators (continuous), as listed earlier (Fig. 1, $\mathrm{H}_{2}$ ). We tested for effect modification by NKB through conducting stratified analysis and by including related interaction terms $(\mathrm{NKB} \times \mathrm{AFHFE})$ in the model (Fig. $\left.1, \mathrm{H}_{6}\right)$. In addition, we assessed confounding by looking at change in estimates between full and reduced models (control for NKB $v$. not) with $10 \%$ as the cut-off used (i.e. a change in estimate $>10 \%$ suggests the existence of confounding) $\left(\text { Fig. 1, } \mathrm{H}_{5}\right)^{(17)}$. A similar criterion was used for mediation by assessing the percentage change in regression coefficients after adjustment was done on the mediating factor (Fig. 1, $\left.\mathrm{H}_{7}\right)^{(18)}$.

All analyses were conducted using survey-related commands in the STATA statistical software package release $9 \cdot 0$ (Stata Corporation, College Station, TX, USA), which takes complex sampling design into account (multistage stratified cluster as opposed to simple random sample) and produces nationally representative estimates of means, proportions and regression coefficients as well as correct estimates of standard errors. In all tests, $P<0.05$ was considered as statistically significant.

\section{Results}

\section{Characteristics of the study sample}

The study participants in the CSFII for the years 1994-1996 ( $n$ 7148) were selected to be in the age range 20 to 65 years (mean $39 \cdot 9$ (SE $0 \cdot 24$ ) years). Overall, $51 \cdot 1 \%$ were female, $18.6 \%$ were non-white, mean number of children under 18 years of age was $0 \cdot 79$ ( SE 0.05), mean years of education was $13 \cdot 3$ (SE $0 \cdot 10)$, mean household income per capita was \$US 16921 (SE \$US 321), employment rate was $55 \cdot 7 \%$ and current smokers accounted for $27 \cdot 5 \%$ of the sample, whereas $32.5 \%$ reported to rarely or never exercise. Within the CSFII/ DHKS group, the proportions of subjects who reported taste, cost, convenience, nutrition and how well food keeps to be very important for them were about $83 \%$, $41 \%, 36 \%, 60 \%$ and $53 \%$, respectively.

\section{Correlates of away-from-bome food expenditure}

Table 1 shows the distribution of selected householdlevel and geographic correlates by weekly absolute and relative AFHFE quintiles. For household-level correlates, mean age of household members increased linearly with absolute AFHFE (38.43v. 34.05 years for upper $v$. lowest quintile, respectively). Similarly, the percentage of females in the household was inversely related to such expenditure without a clear linear trend. On average, the proportion non-white and the mean number of children were also inversely associated with AFHFE. A higher household income per capita, better education attainment and employed status were associated with higher absolute expenditure, with a significant Wilcoxon ranksum test for trend $(P<0 \cdot 05)$. Relative AFHFE exhibited similar associations with these correlates. Regional differentials were noted for absolute but not relative expenditures. In addition, rural areas tended to have households that spent less on away-from-home foods while central city MSA areas and suburban areas were more likely to spend over \$US 15.5 weekly per capita away from home. A proportion of total food cost spent away from home of $33.3 \%$ or more was also more common in central cities and suburban areas, compared with rural areas.

\section{Expenditure in relation to nutrition knowledge and beliefs and diet quality: bivariate analysis}

Table 2 shows unadjusted means of diet quality indicators as well as nutrition belief factors by quintiles of expenditure variables. In terms of dietary intake, means of the selected food groups and nutrients differed significantly 
Table 1 Unadjusted means and proportions, with their standard errors, of household-level and geographic correlates by weekly away-fromhome food expenditure (AFHFE) quintile (absolute and relative): US men and women (20-65 years) participating in the Continuing Survey of Food Intake by Individuals (CSFII) 1994-96

\begin{tabular}{|c|c|c|c|c|c|c|c|c|c|c|}
\hline \multirow[b]{3}{*}{ Household-level correlates } & \multicolumn{10}{|c|}{ Quintile of absolute AFHFE (\$US/capita) (CSFII, $n 7148$ ) } \\
\hline & \multicolumn{2}{|c|}{ Lower $(<1 \cdot 40)$} & \multicolumn{2}{|c|}{ 2nd $(1 \cdot 40-4 \cdot 00)$} & \multicolumn{2}{|c|}{ 3rd $(4 \cdot 00-7 \cdot 75)$} & \multicolumn{2}{|c|}{ 4th $(7 \cdot 75-15 \cdot 50)$} & \multicolumn{2}{|c|}{ Upper $(>15 \cdot 50)$} \\
\hline & \multicolumn{10}{|c|}{ Means with their standard errors } \\
\hline Mean age (years) & 34.05 & $0 \cdot 76$ & $31 \cdot 45$ & 0.58 & $33 \cdot 60$ & 0.57 & $35 \cdot 24$ & $0 \cdot 72$ & $38 \cdot 43^{*}$ & 0.52 \\
\hline$\%$ Female & $49 \cdot 83$ & $1 \cdot 42$ & $50 \cdot 29$ & $1 \cdot 26$ & $47 \cdot 77$ & 0.68 & $48 \cdot 46$ & 0.97 & $45 \cdot 06^{\star}$ & $1 \cdot 12$ \\
\hline$\%$ Non-white & $34 \cdot 63$ & $3 \cdot 20$ & $25 \cdot 69$ & $3 \cdot 17$ & $18 \cdot 50$ & $2 \cdot 73$ & $14 \cdot 62$ & $1 \cdot 80$ & $11 \cdot 96^{*}$ & 1.65 \\
\hline Number of children (age $\leq 18$ years) & 0.79 & 0.05 & 0.72 & 0.04 & 0.56 & 0.04 & 0.39 & 0.04 & $0 \cdot 15^{\star}$ & 0.02 \\
\hline Years of education (age $\geq 20$ years) & $11 \cdot 72$ & $0 \cdot 16$ & $12 \cdot 68$ & $0 \cdot 13$ & $13 \cdot 29$ & $0 \cdot 11$ & $13 \cdot 60$ & $0 \cdot 11$ & $13 \cdot 98^{*}$ & $0 \cdot 11$ \\
\hline Household income/capita (\$US) & 9413 & 456 & 11899 & 323 & 15717 & 287 & 19041 & 480 & $26737^{\star}, \ddagger$ & 526 \\
\hline$\%$ Employed & $39 \cdot 70$ & $2 \cdot 07$ & $54 \cdot 18$ & 1.93 & $59 \cdot 57$ & $1 \cdot 41$ & $60 \cdot 56$ & $1 \cdot 69$ & $67 \cdot 50^{*}$ & $1 \cdot 37$ \\
\hline$\%$ Current smokers & 34.09 & $1 \cdot 87$ & $25 \cdot 19$ & $1 \cdot 30$ & $21 \cdot 19$ & $1 \cdot 28$ & 21.09 & $1 \cdot 50$ & $25 \cdot 51^{*}$ & $1 \cdot 31$ \\
\hline \% Rarely or never exercise & $32 \cdot 51$ & $1 \cdot 75$ & $27 \cdot 76$ & 0.96 & $23 \cdot 07$ & $1 \cdot 12$ & $25 \cdot 06$ & $1 \cdot 41$ & $25 \cdot 90^{\star}$ & 1.46 \\
\hline Geographic correlates & \multicolumn{10}{|c|}{ Proportions with their standard errors } \\
\hline Region & & & & & & & & & & \\
\hline North-east & $21 \cdot 2$ & $2 \cdot 5$ & $17 \cdot 8$ & 0.9 & $17 \cdot 0$ & $2 \cdot 1$ & $18 \cdot 2$ & $1 \cdot 8$ & $25 \cdot 7^{\star}$ & 1.5 \\
\hline Mid-west & $14 \cdot 3$ & $1 \cdot 2$ & $21 \cdot 3$ & $2 \cdot 5$ & $21 \cdot 3$ & 1.5 & $23 \cdot 4$ & $2 \cdot 6$ & $19 \cdot 7$ & $1 \cdot 7$ \\
\hline South & $14 \cdot 5$ & $1 \cdot 1$ & $19 \cdot 4$ & $0 \cdot 7$ & 23.9 & $2 \cdot 2$ & $23 \cdot 2$ & $1 \cdot 4$ & $18 \cdot 9$ & $1 \cdot 8$ \\
\hline West & $18 \cdot 6$ & $2 \cdot 3$ & $20 \cdot 9$ & $2 \cdot 5$ & $20 \cdot 8$ & $2 \cdot 2$ & $18 \cdot 1$ & $2 \cdot 5$ & $21 \cdot 5$ & $2 \cdot 8$ \\
\hline \multicolumn{11}{|l|}{ Urbanization } \\
\hline MSA-central city & $17 \cdot 6$ & $1 \cdot 7$ & $20 \cdot 8$ & 1.5 & $20 \cdot 3$ & 1.5 & $20 \cdot 3$ & 1.9 & $21 \cdot 3+$ & 1.9 \\
\hline MSA-suburban & $14 \cdot 7$ & $1 \cdot 1$ & $16 \cdot 9$ & 0.9 & $22 \cdot 1$ & $1 \cdot 6$ & $22 \cdot 6$ & $1 \cdot 3$ & $23 \cdot 6$ & $1 \cdot 3$ \\
\hline Rural & $19 \cdot 8$ & $1 \cdot 5$ & $25 \cdot 1$ & $2 \cdot 7$ & $21 \cdot 1$ & $1 \cdot 9$ & $19 \cdot 5$ & $1 \cdot 7$ & $14 \cdot 4$ & $1 \cdot 8$ \\
\hline & \multicolumn{10}{|c|}{ Quintile of relative AFHFE (\% of total food expenditure) (CSFII, $n$ 6977§) } \\
\hline & \multicolumn{2}{|c|}{ Lower $(<5 \cdot 75)$} & \multicolumn{2}{|c|}{ 2nd $(5 \cdot 75-13 \cdot 33)$} & \multicolumn{2}{|c|}{ 3rd (13.33-22.02) } & \multicolumn{2}{|c|}{ 4th $(22 \cdot 02-33 \cdot 33)$} & \multicolumn{2}{|c|}{ Upper $(>33 \cdot 33)$} \\
\hline Household-level correlates & \multicolumn{10}{|c|}{ Means with their standard errors } \\
\hline Mean age (years) & 34.97 & 0.73 & $33 \cdot 23$ & 0.52 & $33 \cdot 49$ & 0.52 & $34 \cdot 24$ & 0.61 & $36 \cdot 36^{*}$ & 0.65 \\
\hline$\%$ Female & $49 \cdot 12$ & $1 \cdot 48$ & $48 \cdot 87$ & $1 \cdot 23$ & $48 \cdot 28$ & $1 \cdot 21$ & $48 \cdot 74$ & 0.94 & $46 \cdot 33^{*}$ & $1 \cdot 20$ \\
\hline$\%$ Non-white & $30 \cdot 78$ & $2 \cdot 95$ & 23.69 & $2 \cdot 43$ & $19 \cdot 00$ & 3.03 & $16 \cdot 23$ & $2 \cdot 37$ & $14 \cdot 45^{\star}$ & 1.57 \\
\hline Number of children (age $\leq 18$ years) & 0.67 & 0.05 & 0.66 & 0.04 & 0.54 & 0.04 & 0.46 & 0.04 & $0.28^{*}$ & 0.03 \\
\hline Years of education (age $\geq 20$ years) & $11 \cdot 80$ & $0 \cdot 15$ & $12 \cdot 94$ & $13 \cdot 25$ & $13 \cdot 25$ & $0 \cdot 11$ & $13 \cdot 54$ & $0 \cdot 13$ & $13 \cdot 78^{\star}$ & $0 \cdot 11$ \\
\hline Household income/capita (\$US) & 10663 & 452 & 14368 & 492 & 16627 & 475 & 18523 & 676 & $23058^{*}$ & 563 \\
\hline$\%$ Employed & $41 \cdot 62$ & $2 \cdot 47$ & 55.45 & $1 \cdot 47$ & $58 \cdot 33$ & $1 \cdot 40$ & $61 \cdot 75$ & 1.53 & $66 \cdot 23 t$ & $1 \cdot 44$ \\
\hline$\%$ Current smokers & $33 \cdot 42$ & $2 \cdot 02$ & $27 \cdot 08$ & $1 \cdot 26$ & $21 \cdot 19$ & 1.04 & $21 \cdot 73$ & 1.04 & $23 \cdot 77^{*}$ & $1 \cdot 39$ \\
\hline$\%$ Rarely or never exercise & $32 \cdot 17$ & $1 \cdot 79$ & $25 \cdot 83$ & 0.90 & $25 \cdot 18$ & 1.53 & $24 \cdot 22$ & 1.34 & $25 \cdot 37^{\star}$ & $1 \cdot 43$ \\
\hline Geographic correlates & \multicolumn{10}{|c|}{ Proportions with their standard errors } \\
\hline Region & & & & & & & & & & \\
\hline North-east & $21 \cdot 2$ & $3 \cdot 0$ & $20 \cdot 9$ & 0.9 & $19 \cdot 1$ & $1 \cdot 6$ & $18 \cdot 2$ & $1 \cdot 3$ & $20 \cdot 6$ & $2 \cdot 2$ \\
\hline Mid-west & $15 \cdot \overline{9}$ & $1 \cdot 7$ & $19 \cdot 6$ & $1 \cdot 8$ & $20 \cdot 4$ & 1.5 & $22 \cdot \overline{8}$ & $2 \cdot 2$ & $21 \cdot 2$ & $1 \cdot \overline{5}$ \\
\hline South & $15 \cdot 1$ & $1 \cdot 3$ & $19 \cdot 3$ & $0 \cdot 8$ & $20 \cdot 2$ & $1 \cdot 7$ & $22 \cdot 9$ & $1 \cdot 3$ & $22 \cdot 5$ & $2 \cdot 1$ \\
\hline West & $18 \cdot 1$ & $2 \cdot 5$ & $21 \cdot 4$ & $2 \cdot 1$ & $21 \cdot 0$ & $2 \cdot 3$ & $20 \cdot 0$ & $2 \cdot 9$ & $19 \cdot 4$ & $2 \cdot 1$ \\
\hline \multicolumn{11}{|l|}{ Urbanization } \\
\hline MSA-central city & $17 \cdot 8$ & $1 \cdot 2$ & $20 \cdot 5$ & $1 \cdot 2$ & $19 \cdot 0$ & 1.9 & $21 \cdot 8$ & $1 \cdot 6$ & $20 \cdot 9 t$ & $1 \cdot 8$ \\
\hline MSA-suburban & $15 \cdot 2$ & $1 \cdot 4$ & $18 \cdot 6$ & $1 \cdot 1$ & $20 \cdot 9$ & $1 \cdot 2$ & $22 \cdot 1$ & $1 \cdot 3$ & $23 \cdot 2$ & $1 \cdot 3$ \\
\hline Rural & $20 \cdot 7$ & $1 \cdot 8$ & $23 \cdot 2$ & $1 \cdot 7$ & $20 \cdot 7$ & 1.5 & $18 \cdot 4$ & $1 \cdot 3$ & $16 \cdot 9$ & $1 \cdot 7$ \\
\hline
\end{tabular}

MSA, Metropolitan Statistical Area.

*Wilcoxon rank-sum test for trend: $P<0.05$.

$+\chi^{2}$ test of independence between two categorical variables: $P<0.05$.

$\ddagger$ All $95 \% \mathrm{Cl}$ did not overlap.

§Missing data in the denominator of the relative expenditure variable (total food expenditure) led to a smaller sample size compared with the absolute expenditure variable.

by quintile of expenditure, with few exceptions. Poor dietary intake quality was shown with increased expenditure for all indicators (based on the sign and significance of the Wilcoxon rank-sum test for trend) except for servings of VF, dairy and fibre, which showed the inverse trend (Wilcoxon rank-sum test for trend for absolute expenditure: $z=7 \cdot 82,7 \cdot 97$ and $8 \cdot 38$, respectively, $P<0 \cdot 001)$. Overall, trends in the HEI followed those of VF, dairy and fibre by increasing with higher AFHFE, although only for the absolute expenditure measure. In terms of NKB factors, there was a clear threshold effect at the fifth quintile whereby those with highest expenditure had the poorest mean NKB score, particularly for the global PCA score (absolute expenditure, mean PCA score $=-0.24, P<0.05$ for Wilcoxon rank-sum test; relative expenditure, mean PCA score $=$ $-0 \cdot 30, P<0 \cdot 05)$.

\section{Effect of nutrition knowledge and beliefs on diet quality}

Table 3 presents unadjusted means of dietary intake variables by nutrition belief tertiles and adjusted regression 
Table 2 Unadjusted means with their standard errors of diet quality indicators and indices and nutrition knowledge and belief factors by weekly away-from home-food expenditure (AFHFE) quintile (absolute and relative): US men and women (20-65 years) participating in the Continuing Survey of Food Intake by Individuals (CSFII) and the CSFII/Diet and Health Knowledge Survey (DHKS) 1994-96

\begin{tabular}{|c|c|c|c|c|c|c|c|c|c|c|}
\hline & \multicolumn{10}{|c|}{ Quintile of absolute AFHFE (\$US/capita) } \\
\hline & \multicolumn{10}{|c|}{ CSFII 1994-96 ( $n$ 7148) } \\
\hline & \multicolumn{2}{|c|}{ Lower $(<1 \cdot 40)$} & \multicolumn{2}{|c|}{ 2nd $(1 \cdot 40-4 \cdot 00)$} & \multicolumn{2}{|c|}{ 3rd $(4 \cdot 00-7 \cdot 75)$} & \multicolumn{2}{|c|}{ 4th $(7 \cdot 75-15 \cdot 50)$} & \multicolumn{2}{|c|}{ Upper $(>15 \cdot 50)$} \\
\hline & Mean & SE & Mean & SE & Mean & SE & Mean & SE & Mean & SE \\
\hline \multicolumn{11}{|l|}{ Diet quality indicators and indices } \\
\hline New HEl score & $48 \cdot 9$ & $0 \cdot 6$ & $49 \cdot 8$ & 0.5 & $50 \cdot 3$ & 0.5 & $50 \cdot 7$ & 0.5 & $49 \cdot 8+$ & 0.5 \\
\hline Total fat $(\mathrm{g})$ & $76 \cdot 6$ & $5 \cdot 8$ & $76 \cdot 3$ & $1 \cdot 6$ & $74 \cdot 8$ & $1 \cdot 2$ & $77 \cdot 8$ & $1 \cdot 6$ & $80 \cdot 9+$ & $1 \cdot 3$ \\
\hline Total fat (\% of energy) & $33 \cdot 7$ & $0 \cdot 2$ & $33 \cdot 5$ & 0.3 & $33 \cdot 0$ & $0 . \overline{3}$ & $32 \cdot 9$ & 0.3 & $33 \cdot 3$ & 0.3 \\
\hline Saturated fat $(g)$ & $26 \cdot 4$ & $2 \cdot 5$ & $25 \cdot 4$ & $0 \cdot 6$ & $25 \cdot 1$ & $0 \cdot 4$ & $25 \cdot 9$ & 0.5 & $27 \cdot 1+$ & 0.5 \\
\hline Saturated fat ( $\%$ of energy) & $11 \cdot 3$ & $0 \cdot 2$ & $11 \cdot 1$ & $0 \cdot 1$ & $11 \cdot 0$ & $0 \cdot 1$ & $10 \cdot 9$ & $0 \cdot 1$ & $11 \cdot 1$ & $0 \cdot 1$ \\
\hline Cholesterol (mg) & 288 & 18 & 270 & 6 & 271 & 7 & 269 & 5 & 267 & 6 \\
\hline $\mathrm{Na}(\mathrm{mg})$ & 3391 & 217 & 3398 & 62 & 3392 & 55 & 3527 & 65 & $3578 \dagger$ & \\
\hline Fibre $(\mathrm{g})$ & $14 \cdot 6$ & 0.4 & $15 \cdot 7$ & $0 \cdot 4$ & $15 \cdot 6$ & 0.3 & $16 \cdot 2$ & 0.4 & $16 \cdot 1 \dagger$ & $0 \cdot 2$ \\
\hline Added sugars (teaspoons) & $18 \cdot 0$ & $1 \cdot 4$ & $19 \cdot 3$ & $0 \cdot 4$ & $19 \cdot 0$ & 0.5 & $19 \cdot 2$ & $0 \cdot 6$ & $18 \cdot 7 \dagger$ & $0 \cdot 6$ \\
\hline Sweetened beverages (g) & $309 \cdot 1$ & $17 \cdot 4$ & $347 \cdot 4$ & $16 \cdot 7$ & $344 \cdot 7$ & $17 \cdot 9$ & 339.9 & $21 \cdot 0$ & $328 \cdot 2$ & $18 \cdot 3$ \\
\hline VF (servings) & $4 \cdot 3$ & $0 \cdot 1$ & $4 \cdot 7$ & $0 \cdot 1$ & $4 \cdot 8$ & $0 \cdot 1$ & $5 \cdot 1$ & $0 \cdot 1$ & $5 \cdot 1+$ & $0 \cdot 1$ \\
\hline \multirow[t]{4}{*}{ Dairy products (servings) } & $1 \cdot 3$ & $0 \cdot 1$ & $1 \cdot 3$ & $0 \cdot 0$ & $1 \cdot 3$ & $0 \cdot 0$ & $1 \cdot 4$ & $0 \cdot 0$ & $1 \cdot 4+$ & $0 \cdot 0$ \\
\hline & \multicolumn{10}{|c|}{ CSFII/DHKS 1994-96 ( $n$ 4252) } \\
\hline & \multicolumn{2}{|c|}{ Lower $(<1 \cdot 32)$} & \multicolumn{2}{|c|}{ 2nd $(1 \cdot 32-4 \cdot 00)$} & \multicolumn{2}{|c|}{ 3rd $(4 \cdot 00-7 \cdot 75)$} & \multicolumn{2}{|c|}{ 4th $(7 \cdot 91-16 \cdot 50)$} & \multicolumn{2}{|c|}{ Upper $(>16 \cdot 50)$} \\
\hline & Mean & SE & Mean & SE & Mean & SE & Mean & SE & Mean & SE \\
\hline \multicolumn{11}{|l|}{ Nutrition knowledge/belief factors* } \\
\hline Overall nutrition belief PCA score & $0 \cdot 12$ & $0 \cdot 15$ & -0.08 & $0 \cdot 10$ & -0.06 & 0.09 & $-0 \cdot 10$ & $0 \cdot 11$ & $-0.24 t$ & $0 \cdot 10$ \\
\hline Salt and $\mathrm{Na}$ & $3 \cdot 19$ & 0.06 & $3 \cdot 22$ & 0.05 & $3 \cdot 28$ & 0.04 & $3 \cdot 27$ & 0.05 & $3 \cdot 21$ & 0.04 \\
\hline Saturated fat & $3 \cdot 31$ & 0.05 & $3 \cdot 34$ & 0.04 & $3 \cdot 41$ & 0.03 & 3.38 & 0.05 & $3 \cdot 37$ & 0.03 \\
\hline VF & $3 \cdot 61$ & 0.04 & $3 \cdot 61$ & 0.03 & 3.57 & 0.03 & $3 \cdot 57$ & 0.04 & $3 \cdot 50 t$ & 0.05 \\
\hline Sugars & $3 \cdot 29$ & 0.04 & $3 \cdot 33$ & 0.04 & $3 \cdot 33$ & 0.03 & $3 \cdot 32$ & 0.04 & $3 \cdot 33$ & 0.05 \\
\hline Adequate fibre & $3 \cdot 28$ & 0.05 & $3 \cdot 35$ & 0.03 & $3 \cdot 34$ & 0.04 & $3 \cdot 36$ & 0.04 & $3 \cdot 34$ & 0.03 \\
\hline Variety & $3 \cdot 45$ & 0.04 & $3 \cdot 48$ & 0.04 & 3.53 & 0.04 & 3.53 & 0.03 & $3 \cdot 49$ & 0.03 \\
\hline Healthy weight & 3.65 & 0.04 & $3 \cdot 68$ & 0.03 & $3 \cdot 69$ & 0.03 & $3 \cdot 67$ & 0.03 & $3 \cdot 61$ & 0.03 \\
\hline Fat & 3.46 & 0.04 & $3 \cdot 46$ & 0.04 & $3 \cdot 45$ & 0.04 & $3 \cdot 41$ & 0.04 & $3 \cdot 38$ & 0.03 \\
\hline Cholesterol & $3 \cdot 44$ & 0.04 & $3 \cdot 37$ & 0.05 & $3 \cdot 41$ & 0.04 & $3 \cdot 42$ & 0.03 & $3 \cdot 33$ & 0.03 \\
\hline Breads, cereals, rice, and pasta & 3.00 & 0.04 & $2 \cdot 99$ & 0.05 & 3.06 & 0.03 & 3.06 & 0.04 & 3.00 & 0.04 \\
\hline Milk and dairy products & 3.05 & 0.06 & $3 \cdot 10$ & 0.04 & 3.03 & 0.04 & 3.02 & 0.04 & $2 \cdot 93+$ & 0.05 \\
\hline
\end{tabular}

\begin{tabular}{|c|c|c|c|c|c|c|c|c|c|c|}
\hline & \multicolumn{10}{|c|}{ CSFII 1994-96 (n 6977) } \\
\hline & \multicolumn{2}{|c|}{ Lower $(<5 \cdot 75)$} & \multicolumn{2}{|c|}{ 2nd $(5 \cdot 75-13 \cdot 33)$} & \multicolumn{2}{|c|}{ 3rd (13·33-22·02) } & \multicolumn{2}{|c|}{ 4th $(22 \cdot 02-33 \cdot 33)$} & \multicolumn{2}{|c|}{ Upper $(>33 \cdot 33)$} \\
\hline & Mean & SE & Mean & SE & Mean & SE & Mean & SE & Mean & SE \\
\hline \multicolumn{11}{|l|}{ Diet quality indicators and indices } \\
\hline New HEI score & $49 \cdot 3$ & $0 \cdot 6$ & $50 \cdot 4$ & 0.5 & $51 \cdot 0$ & $0 \cdot 4$ & $49 \cdot 8$ & $0 \cdot 6$ & $49 \cdot 0$ & 0.5 \\
\hline Total fat $(\mathrm{g})$ & $77 \cdot 0$ & $5 \cdot 7$ & $76 \cdot 3$ & $1 \cdot 4$ & $75 \cdot 7$ & $1 \cdot 7$ & $76 \cdot 6$ & $1 \cdot 3$ & $81 \cdot 1+$ & $1 \cdot 2$ \\
\hline Total fat (\% of energy) & $33 \cdot 6$ & 0.3 & $33 \cdot 2$ & $0 \cdot 2$ & $32 \cdot 8$ & $0 \cdot 3$ & $33 \cdot 1$ & 0.3 & $33 \cdot 5$ & 0.2 \\
\hline Saturated fat $(\mathrm{g})$ & $26 \cdot 5$ & $2 \cdot 5$ & $25 \cdot 5$ & 0.5 & $25 \cdot 0$ & $0 \cdot 6$ & $25 \cdot 8$ & 0.5 & $27 \cdot 1+$ & 0.4 \\
\hline Saturated fat ( $\%$ of energy) & $11 \cdot 3$ & 0.2 & $11 \cdot 1$ & $0 \cdot 1$ & $10 \cdot 8$ & $0 \cdot 1$ & $11 \cdot 1$ & $0 \cdot 1$ & $11 \cdot 1$ & $0 \cdot 1$ \\
\hline Cholesterol (mg) & 287 & 17 & 271 & 5 & 264 & 7 & 267 & 7 & 274 & 5 \\
\hline $\mathrm{Na}(\mathrm{mg})$ & 3399 & 212 & 3443 & 62 & 3454 & 63 & 3413 & 49 & $3599+$ & 52 \\
\hline Fibre $(g)$ & $15 \cdot 0$ & 0.5 & $16 \cdot 1$ & 0.4 & $16 \cdot 2$ & $0 \cdot 4$ & $15 \cdot 5$ & $0 \cdot 3$ & $15 \cdot 7 \dagger$ & 0.3 \\
\hline Added sugars (teaspoons) & $18 \cdot 0$ & $1 \cdot 4$ & $19 \cdot 3$ & 0.5 & $18 \cdot 6$ & 0.5 & $19 \cdot 1$ & $0 \cdot 4$ & $19 \cdot 4+$ & 0.5 \\
\hline Sweetened beverages (g) & $312 \cdot 9$ & $19 \cdot 3$ & $340 \cdot 6$ & $19 \cdot 5$ & $318 \cdot 8$ & $16 \cdot 6$ & $343 \cdot 8$ & $16 \cdot 5$ & $357 \cdot 3$ & $21 \cdot 0$ \\
\hline VF (servings) & $4 \cdot 3$ & $0 \cdot 1$ & $5 \cdot 0$ & $0 \cdot 1$ & 4.9 & $0 \cdot 1$ & $4 \cdot 8$ & $0 \cdot 1$ & $5 \cdot 0+$ & $0 \cdot 1$ \\
\hline Dairy products (servings) & $1 \cdot 3$ & $0 \cdot 1$ & $1 \cdot 3$ & $0 \cdot 0$ & $1 \cdot 3$ & $0 \cdot 0$ & $1 \cdot 4$ & $0 \cdot 1$ & $1 \cdot \cdot 4 t$ & 0.0 \\
\hline
\end{tabular}

CSFII/DHKS 1994-96 ( $n$ 4148)

\begin{tabular}{|c|c|c|c|c|c|c|c|c|c|c|}
\hline & \multicolumn{2}{|c|}{ Lower $(<5 \cdot 41)$} & \multicolumn{2}{|c|}{ 2nd (5.41-13.07) } & \multicolumn{2}{|c|}{ 3rd (13.07-21.77) } & \multicolumn{2}{|c|}{ 4th $(21 \cdot 77-33 \cdot 33)$} & \multicolumn{2}{|c|}{ Upper (>33.33) } \\
\hline & Mean & SE & Mean & $\mathrm{SE}$ & Mean & SE & Mean & SE & Mean & SE \\
\hline Vutrition knowledg & & & & & & & & & & \\
\hline $\begin{array}{l}\text { Nutrition knowledge/belief PCA score } \\
\text { Salt and } \mathrm{Na}\end{array}$ & $\begin{array}{l}0 \cdot 16 \\
3 \cdot 24\end{array}$ & $\begin{array}{l}0 \cdot 17 \\
0 \cdot 06\end{array}$ & $\begin{array}{l}0 \cdot 00 \\
3 \cdot 24\end{array}$ & $\begin{array}{l}0 \cdot 10 \\
0.04\end{array}$ & $\begin{array}{r}-0 \cdot 15 \\
3 \cdot 20\end{array}$ & $\begin{array}{l}0.08 \\
0.04\end{array}$ & $\begin{array}{r}-0.08 \\
3 \cdot 32\end{array}$ & $\begin{array}{l}0.08 \\
0.03\end{array}$ & $\begin{array}{c}-0 \cdot 30+ \\
3 \cdot 20\end{array}$ & $\begin{array}{l}0 \cdot 11 \\
0 \cdot 04\end{array}$ \\
\hline
\end{tabular}


CSFII/DHKS 1994-96 (n 4148)

\begin{tabular}{|c|c|c|c|c|c|c|c|c|c|c|}
\hline & \multicolumn{2}{|c|}{ Lower $(<5 \cdot 41)$} & \multicolumn{2}{|c|}{ 2nd (5.41-13.07) } & \multicolumn{2}{|c|}{$3 r d(13 \cdot 07-21 \cdot 77)$} & \multicolumn{2}{|c|}{ 4th $(21 \cdot 77-33 \cdot 33)$} & \multicolumn{2}{|c|}{ Upper (>33.33) } \\
\hline & Mean & SE & Mean & SE & Mean & SE & Mean & SE & Mean & SE \\
\hline Saturated fat & $3 \cdot 32$ & 0.06 & 3.39 & 0.03 & $3 \cdot 34$ & 0.04 & $3 \cdot 46$ & 0.04 & $3 \cdot 31$ & 0.04 \\
\hline VF & $3 \cdot 61$ & 0.04 & 3.62 & 0.03 & 3.56 & 0.03 & 3.57 & 0.05 & $3.50 t$ & 0.05 \\
\hline Sugars & $3 \cdot 31$ & 0.04 & $3 \cdot 38$ & 0.04 & $3 \cdot 28$ & 0.03 & $3 \cdot 35$ & 0.03 & $3 \cdot 29$ & 0.05 \\
\hline Adequate fibre & $3 \cdot 31$ & 0.06 & $3 \cdot 37$ & 0.04 & $3 \cdot 32$ & 0.03 & $3 \cdot 36$ & 0.03 & $3 \cdot 33$ & 0.04 \\
\hline Variety & $3 \cdot 48$ & 0.04 & 3.50 & 0.05 & $3 \cdot 50$ & 0.02 & 3.53 & 0.03 & $3 \cdot 47$ & 0.03 \\
\hline Healthy weight & $3 \cdot 66$ & 0.04 & $3 \cdot 68$ & 0.02 & $3 \cdot 69$ & 0.03 & $3 \cdot 66$ & 0.03 & $3 \cdot 63$ & 0.03 \\
\hline Fat & 3.45 & 0.04 & $3 \cdot 47$ & 0.04 & $3 \cdot 43$ & 0.03 & $3 \cdot 44$ & 0.04 & $3 \cdot 35$ & 0.03 \\
\hline Cholesterol & $3 \cdot 44$ & 0.04 & 3.39 & 0.04 & $3 \cdot 36$ & 0.04 & $3 \cdot 44$ & 0.03 & $3 \cdot 33+$ & 0.03 \\
\hline Breads, cereals, rice, and pasta & $3 \cdot 00$ & 0.05 & 3.04 & 0.04 & 3.06 & 0.03 & $2 \cdot 98$ & $0 \cdot .04$ & $3 \cdot 01$ & 0.05 \\
\hline Milk and dairy products & 3.05 & 0.07 & $3 \cdot 10$ & 0.05 & $2 \cdot 98$ & 0.05 & 3.09 & 0.04 & $2 \cdot 96+$ & 0.04 \\
\hline
\end{tabular}

HEl, Healthy Eating Index; VF, vegetables and fruit; PCA, principal components analysis.

*Nutrition knowledge and belief scale consisted of eleven questions, initiated by the following cue: 'To you personally, is it very important (score of 4), somewhat important (3), not too important (2), or not at all important (score of 1) to consume these foods or nutrients at appropriate levels?' The nutrition knowledge and belief PCA score for each subject is a weighted average of the standardized $z$ scores of each of the eleven responses to the eleven related questions.

+Wilcoxon rank-sum test for trend: $P<0 \cdot 05$.

coefficients between NKB and diet quality indicators and indices. $t$ Tests indicated that all diet quality indicators, with the exception of dairy products, differed significantly between the lowest and the highest NKB score tertiles $(P<0 \cdot 05)$, in the expected direction. In fact, the poorer the NKB score, the higher the intake of total fat, saturated fat, cholesterol, $\mathrm{Na}$ and added sugars and the lower the intake of $\mathrm{VF}$ and fibre. The test for trend (Wilcoxon rank-sum test $P<0.05$ ) was significant for all diet quality indicators except for dairy products (servings). The same trend of improved diet quality with increased NKB score was found for HEI with a significant linear dose-response relationship (Wilcoxon rank-sum test $P<0 \cdot 05$ ).

Subsequently, NKB tertiles were entered as an ordinal variable $(1=$ lower tertile; $2=$ middle tertile; $3=$ upper tertile) into a multivariate model with diet quality indicators as outcomes. In one model ('reduced model'), control was done on individual, household and geographic correlates, while in another model ('full model') relative expenditure was added in addition to the main exposure (NKB score tertile) and the control variables in the reduced model to assess mediation by AFHFE. Eight models out of twelve yielded statistically significant estimates in the expected direction, where a higher NKB tertile was associated with improved dietary quality. Models with total fat (grams and percentage of energy), saturated fat (grams), cholesterol, $\mathrm{Na}$ and VF suggested mediation by expenditure, with a change in regression coefficient estimate by more than $10 \%$. However, this finding was not replicated for HEI. Similar results in terms of change in estimates of regression coefficients between reduced and full models were obtained in a separate mediation analysis where absolute AFHFE was added to the reduced model.

\section{Effect of expenditure on diet quality: multivariate stratified analysis}

Table 4 presents the results of a multivariate linear regression analysis for association between absolute or relative AFHFE and diet quality indicators. The analysis was conducted on all available data for the CSFII sample as well as the sub-sample with data on NKB factors (CSFII/DHKS) stratified by the PCA nutrition belief score tertiles. For the total CSFII sample ( $n$ 7119), absolute AFHFE was positively related to poor diet quality profile of individuals, even after controlling for potential confounders. In particular, households that spent an additional \$US 10 weekly per capita on away-from-home foods had on average an increase of $1.4 \mathrm{~g}$ in fat, 0.2 percentage points in percentage energy from fat, $0.5 \mathrm{~g}$ of saturated fat, $0 \cdot 1$ percentage points in percentage energy from saturated fat, had $0 \cdot 2 \mathrm{~g}$ less fibre intake and $0 \cdot 4$ points fewer on the HEI $(P<0 \cdot 05)$. Similar results were obtained for relative AFHFE.

The analysis stratified by NKB score tertiles indicated that among subjects participating in the DHKS, poor (the lowest tertile) NKB score, fat and saturated fat (in grams), $\mathrm{Na}$ and cholesterol and dairy products were positively related to both absolute and relative $\operatorname{AFHFE}(P<0 \cdot 05)$. As expected, HEI was inversely related to both absolute and relative expenditures. Those with a good (the uppermost tertile) NKB score showed a significant positive association between AFHFE and fat and saturated fat expressed in grams and as percentage of total energy intake. Our additional analysis, by adding interaction terms $(\mathrm{NKB} \times \mathrm{AFHFE})$ in the main models, indicated the presence of interactions between absolute AFHFE and NKB in the case of fat and saturated fat (in grams), cholesterol, $\mathrm{Na}$, added sugar, VF and dairy product intake. For the interaction between relative expenditure 
Table 3 Unadjusted means with their standard errors of diet quality indicators by tertile of nutrition knowledge and belief (NKB) score and adjusted regression coefficients of the effect of NKB tertile on diet, mediation analysis by relative away-from-home food expenditure (AFHFE): US men and women (20-65 years) participating in the Continuing Survey of Food Intake by Individuals (CSFII) and the CSFII/Diet and Health Knowledge Survey (DHKS) 1994-96

\begin{tabular}{|c|c|c|c|c|c|c|c|c|c|c|c|c|}
\hline & & & \multicolumn{10}{|c|}{ CSFII/DHKS 1994-96 } \\
\hline & & & & & & & NKB sco & tertile & & & & \\
\hline & & & & & & & & & & $\begin{array}{l}\text { Adjuste } \\
\text { KB terti }\end{array}$ & $\begin{array}{l}\text { egression cc } \\
\rightarrow \text { diet, ordin }\end{array}$ & \\
\hline & \multicolumn{2}{|c|}{ CSFII 1994-96 } & \multicolumn{2}{|c|}{ Lower (poor score) } & \multicolumn{2}{|c|}{ Middle (moderate score) } & \multicolumn{2}{|c|}{ Upper (good score) } & \multicolumn{2}{|c|}{ Reduced model } & \multicolumn{2}{|c|}{ Full model (+relative AFHFE } \\
\hline & Mean* & SE & Mean & SE & Mean & $\mathrm{SE}$ & Mean & SE & $\hat{\beta} \ddagger$ & $\mathrm{SE}$ & $\hat{\beta} \S$ & SE \\
\hline New HEI score & $49 \cdot 9$ & $0 \cdot 3$ & $45 \cdot 8$ & $0 \cdot 6$ & $50 \cdot 8 \|$ & 0.5 & $54 \cdot 0 \|,{ }^{\star *}$ & $0 \cdot 4$ & 3.0 & $0 \cdot 3$ & $3 \cdot 0$ & $0 \cdot 3$ \\
\hline Total fat $(\mathrm{g})$ & $77 \cdot 3$ & $1 \cdot 1$ & $86 \cdot 8$ & $2 \cdot 0$ & $77 \cdot 5 \|$ & $2 \cdot 9$ & $69 \cdot 9 \|$, ,** & $1 \cdot 6$ & -3.5 & $1 \cdot 1$ & $-3 \cdot 2$ & $1 \cdot 1$ \\
\hline Total fat (\% of energy) & $33 \cdot 2$ & $0 \cdot 1$ & $34 \cdot 4$ & $0 \cdot 3$ & $33 \cdot 1 \|$ & $0 \cdot 4$ & $32 \cdot 5 \|,{ }^{* *}$ & $0 \cdot 3$ & -0.9 & $0 \cdot 2$ & -0.8 & $0 \cdot 2$ \\
\hline Saturated fat $(\mathrm{g})$ & $26 \cdot 0$ & 0.5 & $29 \cdot 5$ & $0 \cdot 7$ & $25 \cdot 8 \|$ & $1 \cdot 3$ & $23 \cdot 0 \|,{ }^{* *}$ & 0.5 & $-1 \cdot 4$ & $0 \cdot 4$ & $-1 \cdot 29$ & 0.4 \\
\hline Saturated fat ( $\%$ of energy) & $11 \cdot 1$ & $0 \cdot 1$ & $11 \cdot 7$ & $0 \cdot 1$ & $10 \cdot 9 \|$ & $0 \cdot 1$ & $10 \cdot 6 \|,{ }^{\star \star}$ & $0 \cdot 1$ & -0.4 & $0 \cdot 1$ & -0.4 & $0 \cdot 1$ \\
\hline Cholesterol (mg) & 272 & 4 & 296 & 9 & $272 \|$ & 10 & $252 \|,{ }^{* *}$ & 7 & $-8 \cdot 3$ & $6 \cdot 6$ & $-6 \cdot 3$ & $6 \cdot 8$ \\
\hline $\mathrm{Na}(\mathrm{mg})$ & 3461 & 48 & 3773 & 88 & $3475 \|$ & 114 & $3203 \|,{ }^{* *}$ & 55 & $-81 \cdot 9$ & $45 \cdot 9$ & $-72 \cdot 9$ & $45 \cdot 7$ \\
\hline Fibre $(\mathrm{g})$ & $15 \cdot 7$ & 0.2 & $15 \cdot 5$ & $0 \cdot 3$ & $15 \cdot 9$ & 0.4 & $16 \cdot 8 \|,{ }^{* *}$ & $0 \cdot 4$ & $1 \cdot 1 ब$ & $0 \cdot 3$ & $1 \cdot 1 \sigma$ & 0.3 \\
\hline Added sugars (teaspoons) & $18 \cdot 9$ & 0.4 & $22 \cdot 2$ & $0 \cdot 6$ & $18 \cdot 4 \|$ & 0.8 & $15 \cdot 4 \|,{ }^{* *}$ & 0.4 & $-1.8 \%$ & 0.4 & $-1 \cdot 8$ & 0.4 \\
\hline Sweetened beverages $(\mathrm{g})$ & $334 \cdot 8$ & $12 \cdot 0$ & $432 \cdot 7$ & $22 \cdot 4$ & $301 \cdot 6 \|$ & $19 \cdot 2$ & $244 \cdot 7 \|$ & $13 \cdot 3$ & $-49 \cdot 2$ & $10 \cdot 6$ & $-47 \cdot 19$ & $10 \cdot 5$ \\
\hline VF (servings) & $4 \cdot 8$ & 0.0 & $4 \cdot 8$ & $0 \cdot 1$ & $4 \cdot 8$ & $0 \cdot 1$ & $5 \cdot 1 \|,{ }^{* \star}$ & $0 \cdot 1$ & $0 \cdot 1$ & $0 \cdot 1$ & 0.2 & $0 \cdot 1$ \\
\hline Dairy products (servings) & $1 \cdot 3$ & 0.0 & $1 \cdot 4$ & $0 \cdot 0$ & $1 \cdot 4$ & $0 \cdot 1$ & $1 \cdot 3$ & $0 \cdot 0$ & $0 \cdot 0$ & $0 \cdot 0$ & 0.0 & $0 \cdot 0$ \\
\hline
\end{tabular}

$\hat{\beta}$, mean regression coefficient; $\mathrm{HEI}$, Healthy Eating Index; $\mathrm{VF}$, vegetables and fruit.

*Survey-based estimates of mean and SE.

+Nutrition knowledge and belief scale consisted of eleven questions, initiated by the following cue: 'To you personally, is it very important (score of 4), somewhat important (3), not too important (2), or not at all important (score of 1 ) to consume these foods or nutrients at appropriate levels?' In regression models, NKB is entered as an ordinal variable $(1=$ lower tertile, $2=$ middle tertile, $3=$ upper tertile).

$\ddagger$ Adjusted for individual-level variables (age, gender, race, education, employment status, smoking status, sedentary behaviour and perceived importance of food safety, cost, taste, convenience and how well food keeps), household income per capita and number of children ( $\leq 18$ years of age), and geographic variables (region and urbanization).

$\| P<0.05$ for null hypothesis that diet quality-related components do not differ between upper and lower tertile of NKB score, using Student's $t$ test.

**Wilcoxon rank-sum test for trend: $P<0.05$ 
Table 4 Multivariate linear regression analysis of the effect of weekly absolute and relative away-from-home food expenditure (AFHFE) (mean adjusted regression coefficients with their standard errors) on selected diet quality indicators, stratified by nutrition knowledge and belief (NKB) score tertile: US men and women (20-65 years) participating in the Continuing Survey of Food Intake by Individuals (CSFII) and the CSFII/Diet and Health Knowledge Survey (DHKS) 1994-96

\begin{tabular}{|c|c|c|c|c|}
\hline & \multicolumn{2}{|c|}{ Absolute AFHFE (\$US/capita) } & \multicolumn{2}{|c|}{$\begin{array}{l}\text { Relative AFHFE (\% of total } \\
\text { food expenditure) }\end{array}$} \\
\hline & $\hat{\beta}^{*}$ & SE & $\hat{\beta}^{*}$ & SE \\
\hline \multicolumn{5}{|l|}{ CSFII 1994-96† } \\
\hline New HEl score & $-0.08 \S$ & 0.01 & $-0.08 \S$ & 0.01 \\
\hline Total fat $(g)$ & $0 \cdot 14 \S$ & 0.06 & $0 \cdot 11$ & 0.06 \\
\hline Total fat (\% of energy) & $0.02 \S$ & 0.01 & $0.02 \S$ & 0.01 \\
\hline Saturated fat $(\mathrm{g})$ & $0.05 \S$ & 0.02 & 0.04 & 0.02 \\
\hline Saturated fat (\% of energy) & $0.01 \S$ & 0.00 & $0.01 \S$ & 0.00 \\
\hline Cholesterol (mg) & 0.43 & $0 \cdot 22$ & 0.24 & $0 \cdot 21$ \\
\hline $\mathrm{Na}(\mathrm{mg})$ & $1 \cdot 48$ & $2 \cdot 01$ & $1 \cdot 13$ & $2 \cdot 01$ \\
\hline Fibre $(\mathrm{g})$ & $-0.02 \S$ & 0.01 & $-0.02 \S$ & 0.01 \\
\hline Added sugars (teaspoons) & 0.02 & 0.02 & 0.03 & 0.02 \\
\hline Sweetened beverages $(\mathrm{g})$ & 0.73 & 0.52 & $1 \cdot 24 \S$ & 0.46 \\
\hline VF (servings) & 0.00 & 0.00 & 0.00 & 0.00 \\
\hline Dairy products (servings) & -0.00 & 0.00 & -0.00 & 0.00 \\
\hline \multirow{2}{*}{\multicolumn{5}{|c|}{ CSFII/DHKS 1994-96† }} \\
\hline \multirow{2}{*}{\multicolumn{5}{|c|}{$\begin{array}{l}\text { By NKB } \neq \text { PCA score } \\
\text { Lower tertile (poor) }\end{array}$}} \\
\hline & & & & \\
\hline New (HEI) score & $-0.08 \S$ & 0.02 & $-0.06 t$ & 0.02 \\
\hline Total fat $(\mathrm{g})$ & $0 \cdot 28 \S, \|$ & 0.09 & $0.27 \S, \|$ & 0.12 \\
\hline Total fat (\% of energy) & -0.01 & 0.02 & 0.00 & 0.01 \\
\hline Saturated fat $(\mathrm{g})$ & $0 \cdot 12 \S, \|$ & 0.04 & $0.11 \S, \|$ & 0.04 \\
\hline Saturated fat (\% of energy) & 0.01 & 0.01 & 0.01 & 0.01 \\
\hline Cholesterol (mg) & $1 \cdot 31 \S, \|$ & 0.46 & $1.22 \S, \|$ & 0.59 \\
\hline $\mathrm{Na}(\mathrm{mg})$ & $6 \cdot 89 \$, \|$ & 3.38 & $6.52 \S, \|$ & 3.21 \\
\hline Fibre $(\mathrm{g})$ & 0.01 & 0.02 & 0.01 & 0.01 \\
\hline Added sugars (teaspoons) & $0.06 \|$ & 0.03 & $-0.04 \|$ & 0.04 \\
\hline Sweetened beverages (g) & -0.04 & 0.94 & 0.75 & 1.14 \\
\hline VF (servings) & $0.01 \|$ & 0.01 & 0.09 & 0.55 \\
\hline Dairy products (servings) & $0.01 \S, \|$ & 0.00 & $0.00 \|$ & 0.00 \\
\hline \multicolumn{5}{|l|}{ Middle tertile (moderate) } \\
\hline New (HEI) score & $-0.08 \S$ & 0.02 & $-0 \cdot 10 \S$ & 0.02 \\
\hline Total fat $(\mathrm{g})$ & -0.06 & $0 \cdot 10$ & -0.04 & 0.09 \\
\hline Total fat (\% of energy) & 0.02 & 0.02 & 0.03 & 0.02 \\
\hline Saturated fat $(\mathrm{g})$ & 0.00 & 0.04 & -0.00 & 0.04 \\
\hline Saturated fat ( $\%$ of energy) & 0.01 & 0.01 & 0.01 & 0.01 \\
\hline Cholesterol (mg) & 0.58 & $0 \cdot 34$ & 0.42 & 0.43 \\
\hline $\mathrm{Na}(\mathrm{mg})$ & -7.05 & $3 \cdot 92$ & $-5 \cdot 21$ & $3 \cdot 78$ \\
\hline Fibre $(\mathrm{g})$ & -0.01 & 0.00 & $-0.06 \S$ & 0.02 \\
\hline Added sugars (teaspoons) & -0.01 & 0.03 & 0.03 & 0.03 \\
\hline Sweetened beverages $(\mathrm{g})$ & 1.69 & $0 \cdot 88$ & $2 \cdot 41 \S$ & $0 \cdot 86$ \\
\hline VF (servings) & $-0.01 \S$ & 0.00 & -0.01 & 0.00 \\
\hline Dairy products (servings) & -0.00 & 0.00 & -0.00 & 0.00 \\
\hline \multicolumn{5}{|l|}{ Upper tertile (good) } \\
\hline New (HEI) score & -0.06 & 0.03 & $-0.05 t$ & 0.02 \\
\hline Total fat $(\mathrm{g})$ & $0.22 \S$ & $0 \cdot 11$ & $0.24 \S$ & 0.08 \\
\hline Total fat (\% of energy) & $0.06 \S$ & 0.03 & $0.05 \S$ & 0.02 \\
\hline Saturated fat $(\mathrm{g})$ & $0.08 \S$ & 0.04 & $0.09 \S$ & 0.03 \\
\hline Saturated fat (\% of energy) & $0.02 \S$ & 0.01 & $0.02 \S$ & 0.01 \\
\hline Cholesterol (mg) & $-0 \cdot 15$ & 0.50 & -0.02 & $0 \cdot 33$ \\
\hline $\mathrm{Na}(\mathrm{mg})$ & $5 \cdot 05$ & $3 \cdot 19$ & $3 \cdot 78$ & $2 \cdot 31$ \\
\hline Fibre $(g)$ & -0.01 & 0.02 & -0.02 & 0.02 \\
\hline Added sugars (teaspoons) & 0.02 & 0.02 & 0.03 & $0 \cdot 03$ \\
\hline Sweetened beverages (g) & 0.57 & 0.72 & 0.50 & $0 \cdot 60$ \\
\hline VF (servings) & 0.01 & 0.01 & 0.01 & 0.01 \\
\hline Dairy products (servings) & -0.00 & 0.00 & -0.00 & 0.00 \\
\hline
\end{tabular}

$\hat{\beta}$, mean regression coefficient; HEI, Healthy Eating Index; VF, vegetables and fruit.

${ }^{*}$ Controlling for individual-level variables (age, gender, race, education, employment status, smoking status and sedentary behaviour), household income per capita and number of children ( $\leqslant 18$ years of age), and geographic variables (region and urbanization). Two-day average intake data were used. Additional control for food purchase factors was done in the stratified models (CSFII/DHKS).

tSurvey-based estimates of $\hat{\beta}$ and SE.

¥Nutrition knowledge and belief scale consisted of eleven questions, initiated by the following cue: 'To you personally, is it very important (score of 4), somewhat important (3), not too important (2), or not at all important (score of 1 ) to consume these foods or nutrients at appropriate levels?' PCA refers to principal components analysis.

$\S P<0.05$ for null hypothesis that $\beta=0$.

$\| P<0.05$ for null hypothesis that interaction term NKB tertile $\times$ expenditure or NKB $\times$ AFHFE is zero $(\gamma=0)$. 
Table 5 Change in estimate in effect of absolute and relative away-from-home food expenditure (AFHFE) on diet quality indicators between reduced and full model (additionally controlling for nutrition knowledge and beliefs, NKB): US men and women (20-65 years) participating in the Continuing Survey of Food Intake by Individuals (CSFII)/Diet and Health Knowledge Survey (DHKS) 1994-96*, +

\begin{tabular}{|c|c|c|c|c|c|c|}
\hline & \multicolumn{3}{|c|}{ Absolute AFHFE (\$US/capita) } & \multicolumn{3}{|c|}{ Relative AFHFE (\% of total food expenditure) } \\
\hline & \multicolumn{3}{|c|}{ Reduced model: not controlling for NKB } & \multicolumn{3}{|c|}{ Reduced model: not controlling for NKB } \\
\hline & $\hat{\beta}^{\star}$ & SE & $\%$ change in estimate & $\hat{\beta}^{*}$ & SE & $\%$ change in estimate \\
\hline New HEI score & $-0.09 \S$ & 0.02 & 7 & $-0.08 \S$ & 0.01 & 7 \\
\hline Total fat $(\mathrm{g})$ & $0 \cdot 15 \S$ & 0.06 & 7 & $0 \cdot 15 \S$ & 0.07 & 4 \\
\hline Total fat (\% of energy) & 0.01 & 0.01 & 7 & $0.02 \S$ & 0.01 & 4 \\
\hline Saturated fat $(\mathrm{g})$ & $0.07 \S$ & 0.03 & 4 & $0.07 \S$ & 0.03 & 3 \\
\hline Saturated fat (\% of energy) & $0.01 \S$ & 0.00 & 8 & $0.01 \S$ & 0.00 & 5 \\
\hline Cholesterol (mg) & $0.63 \S$ & 0.23 & 1 & 0.51 & $0 \cdot 28$ & 2 \\
\hline $\mathrm{Na}(\mathrm{mg})$ & $2 \cdot 43$ & $2 \cdot 06$ & 4 & $2 \cdot 06$ & $2 \cdot 07$ & 6 \\
\hline Fibre $(\mathrm{g})$ & $-0.03 \S$ & 0.01 & 7 & $-0.03 \S$ & 0.01 & 9 \\
\hline Added sugars (teaspoons) & 0.03 & 0.02 & 2 & 0.04 & 0.02 & 11 \\
\hline Sweetened beverages (g) & $0 \cdot 81$ & 0.59 & 12 & $1 \cdot 38$ & 0.63 & 7 \\
\hline VF (servings) & 0.00 & 0.00 & 14 & 0.00 & 0.00 & 16 \\
\hline Dairy products (servings) & 0.00 & 0.00 & 0 & 0.00 & 0.00 & 8 \\
\hline
\end{tabular}

$\hat{\beta}$, mean regression coefficient; HEI, Healthy Eating Index; VF, vegetables and fruit.

${ }^{*}$ Reduced linear regression models controlled for individual-level variables (age, gender, race, education, employment status, smoking status, sedentary behaviour and food purchase factors), household income per capita; geographic variables (region and urbanization); and food purchase factors (perceived importance of food taste, cost, convenience, safety and how well it keeps).

t $n 4232$ for absolute expenditure models; $n 4128$ for relative expenditure models.

$\ddagger \%$ change in estimate $=\|$ Fulll-IReducedl/IReduced $\times 100 \mid$, with full model (results not shown in the table) including NKB tertiles as additional covariates, entered as an ordinal variable ( $1=$ lower tertile, $2=$ middle tertile, $3=$ upper tertile).

$\S P<0.05$ for null hypothesis that $\beta=0$.

and NKB, statistically significant results were found in models with total and saturated fat (grams), cholesterol, $\mathrm{Na}$, added sugars and dairy product intakes as outcome variables. The study results show that an individual's overall nutritional intake (as measured by the HEI) is not significantly reduced by AFHFE if nutrition is important to the individual. Absolute expenditure was inversely and significantly related to HEI in the lower and middle tertiles of NKB score, whereas the association was not significant among those with higher NKB scores (upper tertile), although the interaction between absolute expenditure and NKB did not reach statistical significance in that case.

Further, we tested if NKB was a confounder in the association between expenditure and dietary intake by testing the changes in the estimate of $\beta$ coefficients of the linear regression models including (full models) and excluding (reduced models) NKB tertiles in the models (Table 5). Our findings suggested appreciable changes in estimates $(>10 \%)$ for six out of twenty-four associations tested between expenditure and dietary intake, while borderline significant changes $(>5 \%)$ were observed among eight other associations, most notably those of the HEI.

\section{Discussion}

To our knowledge, the present study is the first to attempt to test the relationship between AFHFE and dietary quality and to examine the interplay of this association with individuals' knowledge and belief about health and nutrition, using nationally representative data from US adults. The main finding was that greater expenditures on away-from-home foods resulted in poorer diet quality. In multivariate analysis of the total CSFII sample, this association held for many diet quality indicators such as fat, saturated fat and fibre as well as the HEI. The stratified analysis by tertile of NKB score in the CSFII/ DHKS sample indicated effect modification whereby AFHFE had a greater effect among individuals with poor NKB scores, although the patterns for some dietary indicators were not consistent. Relative expenditure acted as an intermediate in the pathway between NKB and dietary intake in the case of saturated fat (grams), cholesterol, $\mathrm{Na}$ and VF.

Another important finding of the present study is that, in general, NKB showed a significant and graded positive association with quality of dietary intake independently of socio-economic, lifestyle and geographic factors. The overall score was also inversely related to absolute and relative AFHFE. The important public health implications include that nutrition and health education remains very important to help promote healthy eating among Americans, although some previous studies indicate that broad national nutrition education campaigns have resulted in limited improvement in Americans' VF and dairy consumption in the past dec$\operatorname{ades}^{(19,20)}$. One may argue that, without these campaigns, Americans' eating patterns might be worse.

Several other smaller-scale studies have focused on actual consumption of foods by individuals in fast-food restaurants or other away-from-home outlets and how 
these affected energy intake, diet quality and BMI or obesity among adults ${ }^{(7,8)}$. In general, they suggested that such consumption negatively affects overall diet quality and ultimately promotes weight gain.

The present study has several strengths. First, it closely examined the influence of household-level AFHFE on the diet quality of individual adults using monetary indicators that were measured on a continuous scale rather than a binary 'away-from-home status' indicator variable. Second, the data used were nationally representative and the analyses took into account the complex sampling design. In addition, we looked at several frequently used individual dietary intake variables as well as an overall measure of dietary quality which was revised based on recent dietary guidelines and recommendations.

The study has its limitations as well. One limitation is that the study is based on cross-sectional data, precluding ascertainment of temporality of associations and thus causality. In fact, there may be selection effects that may explain some of the findings. For instance, subjects who value taste and convenience may choose to spend more on foods away from home, while those who value nutrition may choose to eat at home and may have better nutrition beliefs. Although many of these variables were controlled for in our analysis, one cannot rule out residual confounding in such observational studies. Second, because food expenditure was based on a 3-month time frame, there is potential for measurement errors such as due to seasonality, family travel, and accuracy of recall possibly determined by respondent characteristics. However, measurement error of this household-level variable is unlikely to be dependent on dietary quality at the individual level. Therefore, the estimated effect is expected to be biased towards the null, making the true association potentially stronger if the exposure variable was more accurately measured. Finally, the CSFII/DHKS data are 10 years old and one may be concerned about the relevance of these findings for future research and interventions. To our knowledge, restaurant offerings or consumer behaviours over the past decade were not altered dramatically as indicated by the limited improvement in American adults' $\mathrm{VF}$ and dairy consumption $^{(19-22)}$. This supports the important pubic health implications of the association we found between the three variables (i.e. expenditure, nutrition knowledge/ beliefs and diet quality).

Future studies should assess the effects of economic predictors, such as expenditure on foods and their market price locally or at the regional level, on weight change over time through their effect on diet quality, including energy, energy density and intake of VF. Other environmental and socio-economic factors, such as neighbourhood safety, employment hours and work status of individuals, must be considered as well to have a clearer idea about the mechanisms involved in this relationship.
The main policy implications of the present findings include the potential diet quality-related benefit of encouraging households to shift their dietary budget towards home-prepared foods using items purchased in grocery stores and to make desirable choices when eating out at restaurants, fast-foods outlets and cafeterias or when purchasing food and beverage items in vending machines. As many Americans may find it challenging to avoid eating out due to the nature of their occupation, it will be important to empower them to make better dietary choices by mandating nutrition labelling and promoting healthier food selections for consumers at the restaurant level. Indeed, our study results showed that among those individuals with good (upper tertile) NKB, AFHFE did not statistically significantly reduce their overall HEI score. Enhancing individuals' beliefs regarding the importance of nutrition through population-based intervention programmes can help improve diet quality, partly by reducing AFHFE in the case of unhealthy food choices and partly by empowering people to make desirable food choices away from home whenever convenience is a major barrier. In turn, restaurants would be more likely to offer more nutritious choices as the demand for such products increases.

\section{Acknowledgements}

The study was supported in part by the US Department of Agriculture (2044-05322) and the Johns Hopkins University Center for a Livable Future and Bloomberg School of Public Health. Y.W. was also supported by the NIDDK/ NIH (R01 DK63383).

Conflict of interest: None.

Contributions of each author: M.A.B. - conceptualization, plan of analysis, data management and statistical analysis, write-up of manuscript. L.M.P. - conceptualization, plan of analysis, help with statistical analysis, write-up of parts of the manuscript, revision of the manuscript. Y.W. - conceptualization, plan of analysis, help with statistical analysis, write-up of parts of the manuscript, revision of the manuscript.

\section{References}

1. Glanz K, Basil M, Maibach E, Goldberg J \& Snyder D (1998) Why Americans eat what they do: taste, nutrition, cost, convenience, and weight control concerns as influences on food consumption. J Am Diet Assoc 98, 1118-1126.

2. French SA, Story M \& Jeffery RW (2001) Environmental influences on eating and physical activity. Annu Rev Public Health 22, 309-335.

3. Hayden S, Blissard N, Bhuyan S \& Nayga RM Jr (2004) The Demand for Food Away from Home: Full-Service or Fast Food? Agricultural Economic Report no. AER829. Washington, DC: USDA Economic Research Service.

4. Guthrie JF, Lin BH \& Frazao E (2002) Role of food prepared away from home in the American diet, 1977-78 versus 
1994-96: changes and consequences. J Nutr Educ Behav 34, 140-150.

5. Nielsen SJ, Siega-Riz AM \& Popkin BM (2002) Trends in food locations and sources among adolescents and young adults. Prev Med 35, 107-113.

6. Nielsen SJ, Siega-Riz AM \& Popkin BM (2002) Trends in energy intake in US between 1977 and 1996: similar shifts seen across age groups. Obes Res 10, 370-378.

7. Bowman SA \& Vinyard BT (2004) Fast food consumption of US adults: impact on energy and nutrient intakes and overweight status. J Am Coll Nutr 23, 163-168.

8. Satia JA, Galanko JA \& Siega-Riz AM (2004) Eating at fast-food restaurants is associated with dietary intake, demographic, psychosocial and behavioural factors among African Americans in North Carolina. Public Health Nutr 7 , 1089-1096.

9. Schmidt M, Affenito SG, Striegel-Moore R, Khoury PR, Barton B, Crawford P, Kronsberg S, Schreiber G, Obarzanek E \& Daniels S (2005) Fast-food intake and diet quality in black and white girls: the National Heart, Lung, and Blood Institute Growth and Health Study. Arch Pediatr Adolesc Med 159, 626-631.

10. Befort C, Kaur H, Nollen N, Sullivan DK, Nazir N, Choi WS, Hornberger L \& Ahluwalia JS (2006) Fruit, vegetable, and fat intake among non-Hispanic black and non-Hispanic white adolescents: associations with home availability and food consumption settings. J Am Diet Assoc 106, 367-373.

11. US Department of Agriculture (1998) Data (CD-ROM) and Documentation for the 1994-96 Continuing Survey of Food Intakes by Individuals (CSFI)-Diet and Health Knowledge Survey. National Technical Information Service Accession no. PB98-500457. Washington, DC: USDA.

12. Center for Disease Control and Prevention (2006) National Health and Nutrition Examination Survey. http:// www.cdc.gov/nchs/nhanes.htm

13. McCullough ML, Feskanich D, Rimm EB, Giovannucci EL, Ascherio A, Variyam JN, Spiegelman D, Stampfer MJ \&
Willett WC (2000) Adherence to the Dietary Guidelines for Americans and risk of major chronic disease in men. $A m J$ Clin Nutr 72, 1223-1231.

14. US Department of Agriculture, Center for Nutrition Policy and Promotion (2005) Healthy Eating Index 2005. http:// www.cnpp.usda.gov/Publications/HEI/healthyeatingindex 2005factsheet.pdf

15. Sharma S (1996) Applied Multivariate Techniques. New York: Wiley.

16. Cuzick J (1985) A Wilcoxon-type test for trend. Stat Med 4 , 87-90.

17. Maldonado G \& Greenland S (1993) Simulation study of confounder-selection strategies. Am J Epidemiol 138, 923-936.

18. Ditlevsen S, Christensen U, Lynch J, Damsgaard MT \& Keiding N (2005) The mediation proportion: a structural equation approach for estimating the proportion of exposure effect on outcome explained by an intermediate variable. Epidemiology 16, 114-120.

19. Casagrande SS, Wang Y, Anderson C \& Gary TL (2007) Have Americans increased their fruit and vegetable intake? The trends between 1988 and 2002. Am J Prev Med 32, $257-263$.

20. Kant AK \& Graubard BI (2006) Secular trends in patterns of self-reported food consumption of adult Americans: NHANES 1971-1975 to NHANES 1999-2002. Am J Clin Nutr 84, 1215-1223.

21. Guenther PM, Dodd KW, Reedy J \& Krebs-Smith SM (2006) Most Americans eat much less than recommended amounts of fruits and vegetables. J Am Diet Assoc 106, 1371-1379.

22. Fulgoni V 3rd, Nicholls J, Reed A, Buckley R, Kafer K, Huth P, DiRienzo D \& Miller GD (2007) Dairy consumption and related nutrient intake in African-American adults and children in the United States: continuing survey of food intakes by individuals 1994-1996, 1998, and the National Health And Nutrition Examination Survey 1999-2000. J Am Diet Assoc 107, 256-264.

\section{Appendix 1}

\section{Housebold questionnaire (1994-96 Continuing Survey of Food Intake by Individuals): food-related expenditure questions}

Q1: During the past three months, how much money has this household spent per week or per month at grocery stores, including the store's salad bars, soup bars, delis, etc.? Include purchases made with food stamps.

$$
\begin{aligned}
& \text { \$ I_I I_ I_ I, I_ I I_ I _ I.00 PER WEEK _.... } 1 \\
& \text { \$ I_I_II_I, I_I_I I_I.00 PER MONTH _.... } 2
\end{aligned}
$$

Q2: You said this household spent (Amount in Q1) per (week/month). About how much of this amount, if any, was for non-food items, such as cleaning or paper products, food brought for feeding a pet, or cigarettes? (IF NONE, ENTER ' 0 ')

$$
\begin{aligned}
& \text { \$ I_I I_I I_I, I_I I_I I_I.00 PER WEEK ..... } 1 \\
& \text { \$ I_I I_I I I, I_I I_I I_I.00 PER MONTH _.... } 2
\end{aligned}
$$

Q3: During the past three months, how much money has this household spent per week or per month at specialty stores - such as bakeries, liquor stores, delicatessens, meat markets, vegetable stands, health food stores, and other similar places - when the food was brought into your home? (IF NONE, ENTER '0')

$$
\begin{aligned}
& \text { \$ I_I I_I_I, I_I__ I_I.00 PER WEEK ..... } 1 \\
& \text { \$I_I_II_I, I_I__I_I.00 PER MONTH _.... } 2
\end{aligned}
$$

Q4: During the past three months, how much money has this household spent per week or per month at fast food or carryout places when the food was brought into your home? (IF NONE, ENTER '0')

$$
\begin{aligned}
& \text { \$ I_I I_I I_I, I_I I_I I_I.00 PER WEEK ..... } 1 \\
& \$ \text { I I I I I, I I I I I I.00 PER MONTH } \ldots \ldots \quad 2
\end{aligned}
$$

Q5: During the past three months, what has been this household's usual amount of money spent per week or per month for food bought and eaten away from home? Include food and beverages that never entered your home, i.e. eaten at restaurants, fast food places, cafeterias at work or at school or purchased from vending machines, for all household members. (IF NONE, ENTER ' 0 ')

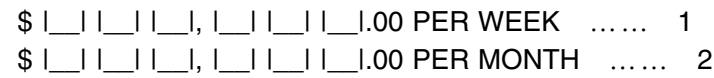


Appendix 2

US Department of Agriculture's new 2005 Healthy Eating Index: components and standards for scoring*

\begin{tabular}{|c|c|c|c|}
\hline Component & Maximum points & $\begin{array}{l}\text { Standard for } \\
\text { maximum score }\end{array}$ & $\begin{array}{l}\text { Standard for } \\
\text { minimum score of } 0\end{array}$ \\
\hline Total fruit (includes $100 \%$ juice) & 5 & $\geq 0.8$ cup equiv. $/ 1000 \mathrm{kcal}$ & No fruit \\
\hline Whole fruit (not juice) & 5 & $\geq 0.4$ cup equiv. $/ 1000 \mathrm{kcal}$ & No whole fruit \\
\hline Total vegetables & 5 & $\geq 1 \cdot 1$ cup equiv. $/ 1000 \mathrm{kcal}$ & No vegetables \\
\hline $\begin{array}{l}\text { Dark green and orange vegetables } \\
\text { and legumest }\end{array}$ & 5 & $\geq 0.4$ cup equiv. $/ 1000 \mathrm{kcal}$ & $\begin{array}{r}\text { No dark green or orange } \\
\text { vegetables or legumes }\end{array}$ \\
\hline Total grains & 5 & $\geq 3.0$ oz equiv./1000 kcal & No grains \\
\hline Whole grains & 5 & $\geq 1.5$ oz. equiv./1000 kcal & No whole grains \\
\hline Milk & 10 & $\geq 1 \cdot 3$ cup equiv. $/ 1000 \mathrm{kcal}$ & No milk \\
\hline Meat and beans & 10 & $\geq 2.5$ oz equiv./1000 kcal & No meat or beans \\
\hline Oils $\S$ & 10 & $\geq 12 \mathrm{~g} / 1000 \mathrm{kcal}$ & No oil \\
\hline Saturated fat & 10 & $\leq 7 \%$ of energyll & $\geq 15 \%$ of energy \\
\hline $\mathrm{Na}$ & 10 & $\leq 0.7 \mathrm{~g} / 1000 \mathrm{kcalll}$ & $\geq 2.0 \mathrm{~g} / 1000 \mathrm{kcal}$ \\
\hline $\begin{array}{l}\text { Energy from solid fat, alcohol, and added } \\
\text { sugar (SoFAAS) }\end{array}$ & 20 & $\leq 20 \%$ of energy & $\geq 50 \%$ of energy \\
\hline
\end{tabular}

Source: reference (14)

To convert kcal to kJ, multiply kcal by $4 \cdot 184$.

*Intakes between the minimum and maximum levels are scored proportionately, except for saturated fat and $\mathrm{Na}$ (see note II).

tLegumes counted as vegetables only after meat and beans standard is met.

fIncludes all milk products, such as fluid milk, yoghurt and cheese.

§Includes non-hydrogenated vegetable oils and oils in fish, nuts and seeds.

IISaturated fat and Na get a score of 8 for the intake levels that reflect the 2005 Dietary Guidelines, $<10 \%$ of energy from saturated fat and $1.1 \mathrm{~g} \mathrm{Na} / 1000 \mathrm{kcal}$, respectively. 